\title{
Efectos de la injuria renal isquémica aguda en la disfunción extrarenal. Estudio experimental aleatorizado
}

\author{
CAMILA SALAS A. ${ }^{1,2}$, PABLO LILLO A. ${ }^{3}$, ALEJANDRO PACHECO V. PHD ${ }^{4}$, TATIANA SALOMÓN S. ${ }^{5}$, \\ FELIPE LILLO A. ${ }^{6}$, ALBERTO GOLDSACK M. ${ }^{6}$, PABLO CRUCES R. ${ }^{1,6}$ \\ 1. Medicina Intensiva Infantil. Hospital Padre Hurtado. Santiago. \\ 2. Becada de Pediatría. Facultad de Medicina Clínica Alemana-Universidad del Desarrollo. Santiago. \\ 3. Escuela de Medicina Veterinaria, Programa de Doctorado en Medicina Veterinaria, Universidad Andrés Bello. Santiago. \\ 4. Tecnólogo Médico. PhD en Ciencias. Departamento de Kinesiología. Universidad Católica del Maule. Talca. \\ 5. Enfermería Intensiva Infantil. UCI Pediátrica, Clínica Alemana de Santiago. \\ 6. Centro de Investigación de Medicina Veterinaria, Escuela de Medicina Veterinaria, Facultad de Ecología y \\ Recursos Naturales, Universidad Andrés Bello. Santiago.
}

\author{
ABSTRACT \\ Acute kidney injury and extrarenal organ dysfunction - A randomized experimental \\ study
}

Background: Acute kidney injury (AKI) is a serious disease that can cause distant organ injuries and is associated with high mortality rates. Objective: To identify the hemodynamic and respiratory dysfunction triggered by AKI, in an animal model of renal ischemia-reperfusion. Method: Twelve anesthetized juvenile pigs $(11.9 \pm 1.0 \mathrm{~kg})$. Six animals underwent ischemia/reperfusion-induced AKI: $45 \mathrm{~min}$ of ischemia and $240 \mathrm{minu}-$ tes of reperfusion, while the remaining six animals were the control group. In basal conditions and after AKI, a conventional hemodynamic monitoring and transpulmonary thermodilution were performed. At the same time, arterial blood gases and lung mechanics were measured. Results: There was a $29 \%$ increase in volumetric preload $\left(221 \pm 22 \mathrm{ml} / \mathrm{m}^{2}\right.$ vs $\left.286 \pm 16 \mathrm{ml} / \mathrm{m}^{2}, \mathrm{p}=0.04\right)$ and a $58 \%$ mean arterial pressure increase $(71 \pm 6 \mathrm{mmHg}$ vs $112 \pm 17 \mathrm{mmHg}, \mathrm{p}=0.04)$ compared to controls, without changes in heart rate, central venous pressure and cardiac output. In addition, an increase of $86 \%$ in extravascular lung water $(7.6 \pm 0.6 \mathrm{ml} / \mathrm{kg} v s 14.1 \pm 1.4 \mathrm{ml} /$ $\mathrm{kg}, \mathrm{p}=0.02$ ) was reported, without changes in gas exchange and lung mechanics. Simultaneously, a slight increase in serum creatinine $(1.12 \pm 0.07 \mathrm{mg} / \mathrm{dl} v s 1.45 \pm 0.10 \mathrm{mg} / \mathrm{dl}, \mathrm{p}=0.03)$ was described. Discussion: An early development of hemodynamic and pulmonary dysfunction was observed in this experimental reperfu-

Recibido el 08 de enero de 2013, devuelto para corregir el 21 de febrero de 2013, segunda versión 02 de marzo de 2013 , aceptado para publicación el 22 de abril de 2013. Este trabajo cumple con los requisitos sobre consentimiento /asentimiento informado, comité de ética, financiamiento, estudios
animales y sobre la ausencia de conflictos de intereses según corresponda.

Estudio financiado por proyecto SOCHIPE 2012001 y FIAC UAB 1102.

Correspondencia a:

Dr. Pablo Cruces R.

E-mail: pcrucesr@gmail.com 
sion model. An increase in volumetric preload and blood pressure associated with a substantial increase in the extravascular lung water were also reported. It is essential serially monitor the hemodynamic and respiratory functions in AKI, including non-uremic subjects.

(Key words: Acute kidney injury, renal ischemia, hemodynamics, pulmonary function).

Rev Chil Pediatr 2013; 84 (3): 268-275

\section{RESUMEN}

Introducción: La insuficiencia renal aguda (IRA) es una enfermedad grave y causante de disfunciones de órganos distantes que incrementan su mortalidad. Objetivo: Identificar disfunción hemodinámica y respiratoria gatillada por IRA en un modelo pediátrico animal de isquemia-reperfusión renal. Método: Doce cerdos anestesiados $(11,9 \pm 1,0 \mathrm{~kg})$. En seis animales se indujo lesión renal bilateral por isquemia-reperfusión: 45 min de isquemia y 240 min de reperfusión, mientras que los seis animales restantes fueron grupo control. En condiciones basales y posterior a lesión renal se realizó monitorización hemodinámica convencional y termodilución transpulmonar. Paralelamente se midió gasometría arterial y análisis de mecánica pulmonar. Resultados: Hubo un incremento de la precarga volumétrica en $29 \%\left(221 \pm 22 \mathrm{ml} / \mathrm{m}^{2} v s 286 \pm 16 \mathrm{ml} / \mathrm{m}^{2}, \mathrm{p}=0,04\right)$ y de la presión arterial media en $58 \%(71 \pm 6 \mathrm{mmHg} v s 112 \pm 17 \mathrm{mmHg}, \mathrm{p}=0,04)$ respecto a controles, sin cambios en frecuencia cardíaca, presión venosa central ni gasto cardíaco. Además ocurrió un aumento del agua extravascular pulmonar en $86 \%(7,6 \pm 0,6 \mathrm{ml} / \mathrm{kg}$ vs $14,1 \pm 1,4 \mathrm{ml} / \mathrm{kg}, \mathrm{p}=0,02)$, sin cambios en intercambio gaseoso ni mecánica pulmonar. Simultáneamente hubo un leve deterioro de la creatinina sérica $(1,12 \pm 0,07 \mathrm{mg} / \mathrm{dL} v \mathrm{~s}$ $1,45 \pm 0,10 \mathrm{mg} / \mathrm{dL}, \mathrm{p}=0,03$ ). Discusión: En este modelo experimental se pudo apreciar el desarrollo temprano de disfunción hemodinámica y pulmonar. Se evidenció un aumento de la precarga volumétrica y de la presión arterial, asociado a un incremento substancial del agua extravascular pulmonar. Creemos que es fundamental monitorizar de forma seriada la función hemodinámica y respiratoria en sujetos con una lesión renal aguda, aún sin una IRA urémica.

(Palabras clave: Insuficiencia renal aguda, isquemia renal, hemodinamia, función pulmonar).

Rev Chil Pediatr 2013; 84 (3): 268-275

\section{Introducción}

La insuficiencia renal aguda (IRA) es una enfermedad devastadora de alta incidencia, caracterizada por una pérdida de la función renal debido a una disminución de la velocidad de filtración glomerular. Esta se traduce en un aumento súbito de los niveles de productos de degradación proteica, sobrecarga hídrica, y alteraciones ácido base e hidroeléctricas de riesgo vital. Adicionalmente, muchos de los pacientes que presentan una IRA desarrollan disfunciones subsecuentes de órganos distantes, desencadenando eventualmente una falla orgánica múltiple, entidad de elevada mortalidad $^{1}$. Gran parte de este riesgo proviene de los efectos de la IRA sobre órganos distantes ${ }^{2-4}$.

Aún no se conocen del todo los mecanismos por los cuales una lesión renal aguda aumenta el riesgo de resultados adversos. En los seres humanos, la determinación de la causalidad es difícil: ¿puede una lesión renal aguda ser causa de morbilidad y mortalidad tan elevadas, o es simplemente un subproducto de la gravedad de la enfermedad subyacente? En este contexto, los modelos animales son de vital importancia a fin de aclarar los mecanismos fisiológicos y probar nuevas terapias ${ }^{4}$. En medicina experimental, la distinción causal es más clara: una lesión renal puede desencadenar efectos deletéreos sistémicos y disfunción de órganos distantes. Nuestro objetivo fue identificar disfunción hemodinámica y respiratoria tempranas en un modelo pediátrico animal de isquemia-reperfusión renal. Este modelo experimental pretende profundizar en el complejo entrecruzamiento entre disfunción de órganos que ocurre después de una lesión renal aguda, los cuales son escasamente conocidos en adultos y niños críticamente enfermos. 


\section{Material y Métodos}

El protocolo experimental fue aprobado por el Comité de Ética Facultad de Medicina Clínica Alemana-Universidad del Desarrollo. Los animales recibieron cuidados en conformidad con los "Guiding Principles in the Care and Use of Laboratory Animals" adoptados por la American Physiological Society.

\section{Sujetos}

Doce cerdos Large-White, menores 2 meses de vida, de $11,9 \pm 1,0 \mathrm{~kg}$. Se estimó el tamaño muestral a través de la comparación de medias independientes, empleando el programa estadístico EPIDAT 2.0. Se asumió un deterioro en la función de órganos distantes de un $30 \%$, con un nivel de confianza de $90 \%$ y una potencia de $80 \%$. Se determinó el empleo de al menos 6 animales por cada grupo experimental.

\section{Grupos experimentales}

Grupo Lesión renal aguda y Grupo Control, ambos con 6 sujetos. Este proceso fue aleatorizado en bloque: de cada 4 animales 2 fueron asignados a cada grupo experimental, pero la secuencia fue producto del azar.

\section{Anestesia}

Se utilizó premedicación con ketamina 15 $\mathrm{mg} / \mathrm{kg}$, midazolam $0,5 \mathrm{mg} / \mathrm{kg}$ y metadona 0,5 $\mathrm{mg} / \mathrm{kg}$ intramuscular seguida por anestesia inhalatoria con isoflurano $1,5 \%$ para los procedimientos iniciales. Durante el resto del protocolo experimental la anestesia fue mantenida con una infusión continua de propofol $(10 \mathrm{mg} /$ $\mathrm{kg} / \mathrm{h})$ y fentanilo $(4 \mu \mathrm{g} / \mathrm{kg} / \mathrm{h})$, adicionándose vecuronio $(0,2 \mathrm{mg} / \mathrm{kg} / \mathrm{h})$ como relajante neuromuscular. La hidratación fue mantenida con solución salina $0,9 \%$ a $10 \mathrm{ml} / \mathrm{kg} / \mathrm{h}$. La temperatura se mantuvo a $37,6 \pm 0,4^{\circ} \mathrm{C}$ usando métodos convectivos convencionales. Un adecuado nivel de anestesia fue asumido si reflejos estaban ausentes.

\section{Protocolo de ventilación}

Se empleó ventilador mecánico EVITA 4 (Dräger Medical, Germany) en modo controlado por volumen con los siguientes parámetros: volumen corriente $\left(\mathrm{V}_{\mathrm{T}}\right) 10 \mathrm{ml} / \mathrm{kg}$, PEEP de $5 \mathrm{~cm} \mathrm{H}_{2} \mathrm{O}$, frecuencia respiratoria (FR) 20 por min, tiempo inspiratorio (TI) $1,0 \mathrm{~s}$ y $\mathrm{FIO}_{2}$ $40 \%$.

\section{Preparación quirúrgica}

La intubación traqueal se realizó con un tubo con cuff de 5,0 $\mathrm{mm}$ de diámetro interno (Mallinckrodt Shiley, St. Louis, MO). La vena yugular externa derecha fue denudada y se instaló un catéter venoso central 7F (Arrow, Reading, $P A, U S A)$, procurando ubicación extra cardíaca. Con técnica similar se instaló catéter arterial $(4 \mathrm{~F})$ con punta termistor en extremo distal (PiCCO ${ }^{\circledR}$ PV2014L08, Pulsion Medical Systems, Munich, Germany) en arteria axilar izquierda y se conectó a monitor $\mathrm{PiCCO}^{\circledR}$ plus para termodilución transpulmonar (TDTP).

\section{Monitorización}

Los signos vitales fueron monitorizados empleando el monitor Infinity Delta XL (Dräger Medical, Germany), registrando la frecuencia cardíaca (FC), presión arterial media (PAM) y presión venosa central (PVC).

\section{Lesión renal aguda}

Posterior a los procedimientos descritos se realizó una laparotomía media. Ambos riñones fueron identificados y disecados libres de tejido circundante y las arterias y venas renales fueron clampeadas sólo en el Grupo Lesión renal aguda. Tras 45 min de isquemia, los clamps vasculares fueron liberados, observándose la inmediata reperfusión renal. El abdomen fue cerrado temporalmente para mantener termorregulación. De los varios modelos animales de lesión renal aguda, la isquemia reperfusión renal es uno de los más comúnmente estudiados porque es reproducible y se puede graduar fácilmente la magnitud de la lesión. Además tiene implicancia clínica en cirugía cardíaca con clampeo aórtico, transplante renal, nefrectomía parcial, paro cardíaco, entre otros ${ }^{5-8}$.

\section{Mediciones}

En condiciones basales y a los $240 \mathrm{~min}$ posterior a la intervención, en ambos grupos experimentales se realizó monitorización hemodinámica convencional y termodilución transpulmonar. 


\section{Medición de gasto cardíaco}

Se realizó TDTP con $5 \mathrm{ml}$ de solución salina $0,9 \%$ fría $\left(<8^{\circ} \mathrm{C}\right)$ por catéter venoso yugular, administradas en forma aleatoria en el ciclo respiratorio. Las mediciones de gasto cardíaco (GC) fueron consideradas válidas cuando las tres medidas aisladas difirieron menos de un $10 \%$ del promedio. En caso de una diferencia $>10 \%$, se realizó una medición adicional. Simultáneamente se consignó precarga volumétrica (GEDV: del inglés global end diastolic volume, volumen diastólico final global) y agua extravascular pulmonar (EVLW: del inglés extravascular lung water). El EVLW es un promisorio parámetro funcional, derivado de termodilución pulmonar, que permite un seguimiento evolutivo del contenido líquido extravascular pulmonar. De este modo, un incremento en el EVLW se interpreta clínicamente como edema pulmonar. Para GEDV se consideraron valores indizados según superficie corporal. Área de superficie corporal fue calculada usando la siguiente fórmula: área de superficie corporal $=\mathrm{K} /$ peso $(\mathrm{en}$ kilogramos) $)^{2,3}$, donde $\mathrm{K}=0,112$ para cerdos 9 . EVLW se expresó como $\mathrm{ml} / \mathrm{kg}$ peso corporal.

\section{Mediciones de gasometría y mecánica pulmonar}

Se midió gasometría arterial y venosa central empleando cartridges i-STAT ${ }^{\circledR} \mathrm{G} 3+($ Abbott Laboratories, Princeton, $N J$ ) al inicio y al finalizar el período experimental, consignándose $\mathrm{PaO}_{2}$ y $\mathrm{PaCO}_{2}$. La presión diferencial fue calculada como la diferencia entre presiones inspiratoria (presión plateau, $\mathrm{P}_{\mathrm{PL}}$ ) y espiratoria final (PEEP total) de las vías aéreas, medidas tras una pausa inspiratoria y espiratoria $4 \mathrm{~s}$ respectivamente. La compliance estática del sistema respiratorio $\left(C_{R S}\right)$ fue calculada como la razón entre el $V_{T}$ espirado y la presión diferencial.

\section{Mediciones de función renal}

La concentración de creatinina sérica se midió utilizando el fotómetro Humalyzer 2000 (Human GmbH, Wiesbaden, Germany). Aún bajo anestesia (Estado 3 - Plano 2), los animales fueron eutanasiados por perfusión de cloruro de potasio al 10\% hasta la detección de la fibrilación ventricular o asistolia.

\section{Análisis estadístico}

Los valores fueron expresados como media \pm SEM. Se utilizó el test Shapiro-Wilk para evaluar la normalidad de las variables. Para comparar las variables estudiadas antes y después de la lesión por isquemia reperfusión renal se utilizó la prueba de Wilcoxon de rangos señalados y pares igualados para dos muestras dependientes. Se consideró significativo un $\mathrm{p}<0,05$. El análisis estadístico se realizó con programa GraphPad Prism $5^{\circledR}$ (San Diego, USA).

\section{Resultados}

Todos los animales completaron el protocolo experimental.

Previo a la lesión renal ambos grupos no diferían en función hemodinámica, respiratoria ni renal (tabla 1). Posterior a la lesión renal se identificaron las alteraciones detalladas en la tabla 2.

\section{Monitorización hemodinámica}

Los sujetos con lesión renal aguda tuvieron un incremento de la PAM $(\mathrm{p}=0,04)$ y del $\operatorname{GEDV}(\mathrm{p}=0,04)$ respecto al grupo control. No se apreciaron cambios significativos en la FC, la PVC ni en el GC entre grupos experimentales.

Tabla 1. Parámetros hemodinámicos, respiratorios y renales en grupos estudiados previo a lesión renal aguda

\begin{tabular}{|c|c|c|}
\hline & Control & LRA \\
\hline FC (latidos por min) & $102 \pm 12$ & $97 \pm 11$ \\
\hline PAM $(\mathrm{mmHg})$ & $88 \pm 8$ & $86 \pm 6$ \\
\hline PVC (mmHg) & $13,7 \pm 1,2$ & $14,3 \pm 1,1$ \\
\hline $\mathrm{GC}(1 / \mathrm{min})$ & $1,21 \pm 0,14$ & $1,07 \pm 0,18$ \\
\hline $\operatorname{GEDV}\left(\mathrm{ml} / \mathrm{m}^{2}\right)$ & $269 \pm 26$ & $243 \pm 16$ \\
\hline $\mathrm{C}_{\mathrm{RS}}\left(\mathrm{ml} / \mathrm{kg} / \mathrm{cmH}_{2} \mathrm{O}\right)$ & $0,77 \pm 0,04$ & $0,70 \pm 0,03$ \\
\hline $\mathrm{PaO}_{2}(\mathrm{mmHg})$ & $171 \pm 6$ & $173 \pm 8$ \\
\hline $\mathrm{PaCO}_{2}(\mathrm{mmHg})$ & $39 \pm 3$ & $43 \pm 3$ \\
\hline $\operatorname{EVLW}(\mathrm{ml} / \mathrm{kg})$ & $10,4 \pm 1,1$ & $9,4 \pm 0,9$ \\
\hline Creatinina plasma $(\mathrm{ml} / \mathrm{dl})$ & $1,13 \pm 0,05$ & $1,13 \pm 0,05$ \\
\hline
\end{tabular}

LRA: lesión renal aguda, FC: frecuencia cardíaca, PAM: presión arterial media, PVC: presión venosa central, GC: gasto cardíaco, GEDV: volumen global diastólico final, $C_{R S}$ : compliance del sistema respiratorio, EVLW: agua extravascular pulmonar. Resultados expresados como media \pm SEM. Todos $p>0,05$. 
Tabla 2. Parámetros hemodinámicos, respiratorios y renales en grupos estudiados $\mathbf{2 4 0}$ min posterior a lesión renal aguda

\begin{tabular}{|c|c|c|}
\hline & Control & LRA \\
\hline FC (latidos por min) & $126 \pm 10$ & $91 \pm 11$ \\
\hline PAM $(\mathrm{mmHg})$ & $71 \pm 6$ & $112 \pm 17^{*}$ \\
\hline PVC (mmHg) & $13,0 \pm 1,0$ & $13,8 \pm 1,9$ \\
\hline $\mathrm{GC}(1 / \mathrm{min})$ & $1,26 \pm 0,17$ & $1,35 \pm 0,16$ \\
\hline $\operatorname{GEDV}\left(\mathrm{ml} / \mathrm{m}^{2}\right)$ & $221 \pm 22$ & $286 \pm 16^{*}$ \\
\hline $\mathrm{C}_{\mathrm{RS}}\left(\mathrm{ml} / \mathrm{kg} / \mathrm{cmH}_{2} \mathrm{O}\right)$ & $0,76 \pm 0,04$ & $0,83 \pm 0,06$ \\
\hline $\mathrm{PaO}_{2}(\mathrm{mmHg})$ & $179 \pm 8$ & $168 \pm 6$ \\
\hline $\mathrm{PaCO}_{2}(\mathrm{mmHg})$ & $46 \pm 2$ & $42 \pm 2$ \\
\hline $\operatorname{EVLW}(\mathrm{ml} / \mathrm{kg})$ & $7,6 \pm 0,6$ & $14,1 \pm 1,4^{*}$ \\
\hline Creatinina plasma (ml/dl) & $1,12 \pm 0,07$ & $1,45 \pm 0,10^{*}$ \\
\hline
\end{tabular}

LRA: lesión renal aguda, FC: frecuencia cardíaca, PAM: presión arterial media, PVC: presión venosa central, GC: gasto cardíaco, GEDV: volumen global diastólico final, $C_{R S}$ : compliance del sistema respiratorio, EVLW: agua extravascular pulmonar. Resultados expresados como media \pm SEM. Consideramos significativo un $\left.\mathrm{p}<0,05{ }^{*}\right)$

\section{Monitorización respiratoria}

El grupo con lesión renal aguda tuvo un incremento en el EVLW $(p=0,02)$ respecto al grupo control, sin cambios en la $\mathrm{PaO}_{2}$, la $\mathrm{PaCO}_{2}$, ni en la compliance estática.

\section{Monitorización renal}

Al final del experimento los sujetos con lesión renal tuvieron niveles de creatinina sérica mayores que el grupo control $(p=0,03)$.

\section{Discusión}

En este modelo experimental pediátrico de lesión renal aguda secundaria a isquemia reperfusión, los principales hallazgos fueron los siguientes:

1) Ocurrió un incremento de la precarga volumétrica en un $29 \%$ y de la presión arterial media en un $58 \%$.

2) Hubo un aumento concomitante del agua extravascular pulmonar en un $86 \%$ respecto a sujetos no lesionados.

Respecto a los efectos hemodinámicos, nuestros resultados concuerdan parcialmente con lo reportado en la literatura. El síndrome cardiorrenal se caracteriza por la combinación de insuficiencia cardíaca y renal, donde uno de los órganos es el principal responsable de la disfunción concomitante del otro órgano. A pesar de su creciente reconocimiento, es pobremente entendido en la actualidad, existiendo pocos estudios experimentales que aborden las interacciones entre corazón y riñón ${ }^{10}$. En particular, el síndrome cardiorrenal tipo 3 define el desarrollo de insuficiencia cardíaca que se produce posterior a la IRA. Varios mecanismos han sido sugeridos para explicar el desarrollo de disfunción cardíaca después de una lesión renal, incluyendo la sobrecarga de líquidos, vasoconstricción pulmonar secundaria a acidemia, pericarditis urémica y disturbios electrolíticos responsables de arritmias ventriculares ${ }^{11-13}$. La ecocardiografía cardíaca en ratones a las $48 \mathrm{~h}$ de una lesión renal isquémica, mostró dilatación ventricular izquierda, aumento del tiempo de relajación ventricular, y la disminución de la fracción de acortamiento ${ }^{14}$. Dentro de los resultados en nuestro modelo de isquemia-reperfusión renal, identificamos un aumento del GEDV a sólo 4 h de la lesión renal, concordante con la dilatación cardíaca previamente descrita. Si bien hubo un incremento significativo de la creatinina sérica en igual período, este fue de muy poca relevancia. En otras palabras, la disfunción hemodinámica fue identificada previo al establecimiento del "resultado funcional" de insuficiencia renal establecida. Estas alteraciones probablemente sean atribuibles a la respuesta inflamatoria desencadenada por la isquemia-reperfusión renal más que a la uremia. No apreciamos una disminución del gasto cardíaco, lo que atribuimos a la corta duración del modelo.

El desarrollo temprano de hipertensión arterial es un hallazgo de mucho interés y estaría estrechamente relacionado a la oxigenación regional. El impacto de los factores hemodinámicos sobre la oxigenación renal permanece lejos de ser comprendido a cabalidad. Estos "factores hemodinámicos" comprenden fundamentalmente a la PAM, el flujo sanguíneo renal (FSR) y su distribución en el territorio vascular cortical y medular, y la tasa de filtración glomerular (TFG). Estos factores son fundamentales para comprender no sólo la fi- 
siología de la oxigenación regional, sino también las causas y consecuencias de la desregulación del flujo regional en la enfermedad renal. Además, tras una lesión renal aguda ocurren múltiples respuestas moleculares y celulares en el tejido renal. Dada su condición de órgano multifuncional, tras una lesión renal aguda, el control de volumen de fluido intravascular, la producción de eritrocitos, y la función endocrina pueden verse alteradas ${ }^{15-17}$. La presión arterial en sí probablemente tiene poco impacto directo en la oxigenación renal. Así, cuando la presión arterial cae dentro del rango de autorregulación, tanto el FSR y como la TFG pueden ser mantenidas por una combinación de dilatación arteriolar aferente y constricción arteriolar eferente. A la inversa, el FSR y la TFG pueden ser mantenidos frente a la presión arterial elevada mediante una combinación de constricción arteriolar aferente y dilatación arteriolar eferente ${ }^{18,19}$. Creemos que la hipertensión de inicio precoz que apreciamos en nuestro modelo experimental pudiera reflejar un mecanismo neuroendocrino (probablemente poco eficaz) que busca preservar el FSR tras pérdida de la autorregulación del flujo regional secundario a la lesión inducida, de manera similar a lo que ocurre en el síndrome de hipertensión intracraneal. Futuros estudios debieran dilucidar esta hipótesis.

Respecto a la disfunción respiratoria, pudimos evidenciar un incremento marcado del EVLW. Este resultado no es sorprendente, dado que las complicaciones respiratorias se asocian frecuentemente a IRA. Sobre la base de la asociación observada entre la IRA y la lesión pulmonar en los seres humanos, los primeros trabajos en modelos animales demostraron que la lesión renal primaria podría conducir a la lesión pulmonar secundaria, fenómeno conocido como injuria pulmonar inducida por una lesión renal aguda (del inglés $A K I$-induced lung injury) ${ }^{13,20,21}$. Se ha demostrado previamente que en forma precoz en una lesión renal aguda hay un incremento de Interleuquina-6 (IL-6), una citoquina que induce migración de neutrófilos hacia el tejido pulmonar y fuga de fluidos hacia el compartimento extravascular pulmonar, con el consiguiente desarrollo de edema pulmonar ${ }^{22}$. La respuesta inflamatoria no controlada después de una lesión renal aguda conduce a un aumento de la permeabilidad vascular pulmonar, como se demostró por la extravasación pulmonar del colorante azul de Evans a las 24 y $48 \mathrm{~h}$ en modelo murino de isquemia-reperfusión renal ${ }^{20}$. El deterioro de la integridad vascular induce la acumulación de líquido en el tejido intersticial pulmonar, dando lugar a edema pulmonar ${ }^{4,23}$. Además, la extravasación de líquido en los alvéolos inactiva el surfactante, causando aún más deterioro de la compliance ${ }^{23}$, pudiendo incrementar el trabajo respiratorio espontáneo y contribuyendo al desarrollo de la insuficiencia respiratoria aguda $^{24}$. Nosotros no identificamos deterioro de mecánica pulmonar ni alteraciones en el intercambio gaseoso, probablemente debido a la corta duración del modelo. El mecanismo intrínseco compensatorio del edema pulmonar intersticial depende de la actividad de la bomba sodio-potasio, con la difusión pasiva de agua a través de canales de acuaporina. Sin embargo, una lesión renal aguda isquémica no sólo causa edema pulmonar intersticial, sino que también regula a la baja tanto a la bomba de sodio y potasio como a la acuaporina, anulando efectivamente este mecanismo de compensación ${ }^{20,25}$. Concordantemente los modelos animales con actividad acuaporina reducida demuestran una predisposición para la lesión pulmonar inducida por ventilación mecánica ${ }^{26}$.

Las principales limitaciones de este estudio fueron las siguientes:

1. Dado que este es un modelo de corta duración, la magnitud de las disfunciones orgánicas probablemente subestiman las repercusiones posibles en niños críticamente enfermos, donde la IRA persiste por muchos días.

2. Consideramos sólo las consecuencias hemodinámicas y respiratorias bajo una condición previa de función cardiovascular y pulmonar normal, siendo conocido que los efectos de una lesión renal aguda son más acentuados en sujetos con disfunciones previas $^{3}$, frecuentes en niños gravemente enfermos. Dadas las limitaciones mencionadas, la extrapolación de estos resultados al quehacer clínico debe ser cuidadosa.

3. Tradicionalmente se ha utilizado la termo- 
dilución pulmonar (catéter de arteria pulmonar) para monitorización hemodinámica avanzada. Sin embargo, el empleo de TDTP está suficientemente validado en pacientes y modelos pediátricos en la actualidad ${ }^{27-29}$. TPTD nos permitió cuantificar de forma seriada precarga volumétrica y agua extravascular pulmonar, no siendo esto posible con termodilución pulmonar. En concordancia, nuestros hallazgos más relevantes fueron derivados de TDTP, siendo escasamente detectados por monitorización convencional.

A pesar de las limitaciones mencionadas nos parece de gran importancia monitorizar de forma seriada la función hemodinámica y respiratoria en pacientes con una lesión renal aguda, aunque estas no sean una IRA "urémi$c a$ ”. Esto es fundamental si consideramos que las disfunciones orgánicas desencadenadas por un lesión renal aguda son potencialmente reversibles.

\section{Referencias}

1.- Nguyen M, Bissler J: Chapter 104. Acute Renal Failure. En Pediatric Critical Care Medicine. Basic Science and Clinical Evidence. Springer-Verlag London Limited 2007. Pag 1221-7.

2.- De Mendona A, Vincent JL, Suter PM, et al: Acute renal failure in the ICU: Risk factors and outcome evaluated by the SOFA score. Intensive Care Med 2000; 26: 915 21.

3.- Levy EM, Viscoli CM, Horwitz RI: The effect of acute renal failure on mortality. A cohort analysis. JAMA 1996; 275: 1489-94.

4.- Yap SC, Lee HT: Acute kidney injury and extrarenal organ dysfunction: new concepts and experimental evidence. Anesthesiology 2012; 116: 1139-48.

5.- Kashyap VS, Cambria RP, Davison JK, L'Italien GJ: Renal failure after thoracoabdominal aortic surgery. J Vasc Surg 1997; 26: 949-55.

6.- Shekarriz B, Shah G, Upadhyay J: Impact of temporary hilar clamping during laparoscopic partial nephrectomy on postoperative renal function: A prospective study. J Urol 2004; 172: 54-7.

7.- Tilney NL, Guttmann RD: Effects of initial ischemia/ reperfusión injury on the transplanted kidney. Transplantation 1997; 64: 945-7.
8.- Ovalle P, Vogel A, Córdova G, Cerda J, Cavagnaro F: Reemplazo renal en el post-operatorio de niños sometidos a cirugía cardíaca con circulación extracorpórea. Rev Chil Pediatr 2012; 83: 24-32.

9.- Roch A, Michelet P, Lambert D, et al: Accuracy of the double indicator method for measurement of extravascular lung water depends on the type of acute lung injury. Crit Care Med 2004; 32: 811-7.

10.- Ronco C, Haapio M, House AA, et al: Cardiorenal syndrome. J Am Coll Cardiol 2008; 52: 1527-39.

11.- Ronco C, Chionh CY, Haapio M, Anavekar NS, House A, Bellomo R: The cardiorenal syndrome. Blood Purif 2009; 27: 114-26.

12.- Figueras J, Stein L, Diez V, Weil MH, Shubin H: Relationship between pulmonary hemodynamics and arterial $\mathrm{pH}$ and carbon dioxide tension in critically ill patients. Chest 1976; 70: 466-72.

13.- Grams ME, Rabb H: The distant organ effects of acute kidney injury. Kidney Int 2012; 81: 942-8.

14.- Kelly KJ: Distant effects of experimental renal ischemia/ reperfusion injury. J Am Soc Nephrol 2003; 14: 1549-58.

15.- Evans $R G$, Ince $C$, Joles $J A$, et al: Haemodynamic influences on kidney oxygenation: The clinical implications of integrative physiology. Clin Exp Pharmacol Physiol 2012; En prensa.

16.- Zhang $W$, Edwards A: Oxygen transport across vasa recta in the renal medulla. Am J Physiol Heart Circ Physiol 2002; 283: H1042-55.

17.- Gardiner BS, Smith DW, O'Connor PM, Evans RG: A mathematical model of diffusional shunting of oxygen from arteries to veins in the kidney. Am J Physiol Renal Physiol 2011; 300: F1339-52.

18.- Just A: Mechanisms of renal blood flow autoregulation: dynamics and contributions. Am J Physiol Regul Integr Comp Physiol 2007; 292: R1-17.

19.- Hall JE, Guyton AC, Cowley AW Jr: Dissociation of renal blood flow and filtration rate autoregulation by renin depletion. Am J Physiol 1977; 232: F215-21.

20.- Kramer AA, Postler G, Salhab KF, Méndez C, Carey $L C, R a b b H$ : Renal ischemia/reperfusion leads to macrophage-mediated increase in pulmonary vascular permeability. Kidney Int 1999; 55: 2362-7.

21.- Hoke TS, Douglas IS, Klein CL, et al: Acute renal failure after bilateral nephrectomy is associated with cytokine-mediated pulmonary injury. J Am Soc Nephrol 2007; 18: 155-64.

22.- Klein CL, Hoke TS, Fang WF, Altmann CJ, Douglas IS, Faubel $S$ : Interleukin-6 mediates lung injury following ischemic acute kidney injury or bilateral nephrectomy. Kidney Int 2008; 74: 901-9. 
23.- Miserocchi G, Negrini D, Passi A, De Luca G: Development of lung edema: Interstitial fluid dynamics and molecular structure. News Physiol Sci 2001; 16: 66-71.

24.- Turcios NL: Pulmonary complications of renal disorders. Paediatr Respir Rev 2012; 13: 44-9.

25.- Hassoun HT, Lie ML, Grigoryev DN, Liu M, Tuder RM, Rabb $H$ : Kidney ischemia-reperfusion injury induces caspase-dependent pulmonary apoptosis. Am J Physiol Renal Physiol 2009; 297: F125-37.

26.- Hales CA, Du HK, Volokhov A, Mourfarrej R, Quinn $D A$ : Aquaporin channels may modulate ventilatorinduced lung injury. Respir Physiol 2001; 124: 15966.

27.- Tibby SM, Hatherill M, Marsh MJ, Morrison G, An- derson D, Murdoch IA: Clinical validation of cardiac output measurements using femoral artery thermodilution with direct Fick in ventilated children and infants. Intensive Care Med 1997; 23: 987-91.

28.- Rupérez M, López-Herce J, García C, Sánchez C, García E, Vigil D: Comparison between cardiac output measured by the pulmonary arterial thermodilution technique and that measured by the femoral arterial thermodilution technique in a pediatric animal model. Pediatr Cardiol 2004; 25: 119-23.

29.- Lemson J, de Boode WP, Hopman JC, Singh SK, van der Hoeven $J G$ : Validation of transpulmonary thermodilution cardiac output measurement in a pediatric animal model. Pediatr Crit Care Med 2008; 9: 313-9. 